\title{
Recombinant adeno-associated virus mediated RNA interference inhibits metastasis of nasopharyngeal cancer cells in vivo and in vitro by suppression of Epstein-Barr virus encoded LMP-1
}

\author{
XIANGPING LI ${ }^{1}$, XIONG LIU ${ }^{1}$, CHRISTINE Y.S. LI $^{3}$, YANQING DING $^{1}$, DAVID CHAU $^{3}$, \\ GANG LI ${ }^{1}$, HSIANG-FU KUNG ${ }^{4,5}$, MARIE C.M. LIN ${ }^{3}$ and YING PENG ${ }^{2}$
}

\author{
${ }^{1}$ Department of Otolaryngology, Nanfang Hospital, Nanfang Medical University, Guangzhou 510515; \\ ${ }^{2}$ Department of Neurology, The Second Affiliated Hospital, Sun Yat-Sen University, Guangzhou 510120; \\ ${ }^{3}$ Institute of Molecular Biology and Open Laboratory of Chemical Biology of Institute of Molecular Technology \\ for Drug Discovery, The University of Hong Kong; ${ }^{4} \mathrm{Li} \mathrm{Ka}$ Shing Institute of Health Sciences and ${ }^{5}$ Centre for Emerging \\ Infectious Disease, Faculty of Medicine, The Chinese University of Hong Kong, Hong Kong, P.R. China
}

Received January 18, 2006; Accepted March 9, 2006

\begin{abstract}
Nasopharyngeal carcinoma (NPC) is a highly metastatic carcinoma characterized by consistent association with Epstein-Barr virus (EBV). Of the EBV-encoded product, latent membrane protein-1 (LMP-1) is considered to be an oncoprotein playing an essential role in cell transformation and metastasis. In this study, we used a recombinant adenoassociated virus type 2 vector (rAAV-2) to deliver small hairpin RNA (shRNA) targeting EBV LMP-1 into the EBV-positive human NPC C666-1 cells and evaluated the effect of long-term suppression of LMP-1 on NPC growth and metastasis in vivo and in vitro. An NPC metastasis nude mouse model with NPC xenograft transplanted in liver was established. The NPC C666-1 cells infected with rAAV-shRNA-LMP-1 or rAAVEGFP were inoculated in the livers of nude mice. Formation of liver and lung metastasis was evaluated at day 14 after tumor inoculation. Our results demonstrate that rAAV-shRNA-
\end{abstract}

Correspondence to: Dr Ying Peng, Department of Neurology, The Second Affiliated Hospital, Sun Yat-Sen University, Guangzhou 510120, P.R. China

E-mail: pengy12@yahoo.com

Dr Marie C.M. Lin, Department of Chemistry, 8/F, Kadoorie Biological Sciences Building, The University of Hong Kong, Pokfulam Road, Hong Kong, P.R. China

E-mail: mcllin@hkusua.hku.hk

Abbreviations: rAAV, recombinant adeno-associated virus; NPC, nasopharyngeal carcinoma; LMP-1, latent membrane protein-1; DMSO, dimethyl sulfoxide; PBS, phosphate-buffered solution; shRNA, small hairpin RNA; siRNA, small interference RNA

Key words: rAAV, nasopharyngeal carcinoma, RNAi, LMP-1, metastasis
LMP-1 effectively infected C666-1 cells and suppressed LMP-1 expression. Such suppression, in turn, did not significantly inhibit tumor growth, but prevented NPC metastasis in the liver as well as in the lung. Consistent with in vivo data, the in vitro studies in NPC C666-1 cell cultures showed that suppression of LMP-1 by rAAV-shRNA-LMP-1 significantly reduced cell mobility and transmembrane invasion ability. These results demonstrated for the first time that long-term suppression of EBV-encoded LMP-1 in vivo is an effective means for preventing NPC metastasis.

\section{Introduction}

Nasopharyngeal carcinoma (NPC) is a highly malignant tumor which can easily invade local tissues and metastasize to distant organs, such as cervical lymph nodes, liver, lung, bone, and brain. It has been reported that over $60 \%$ of NPC patients already have clinically detectable metastasis in many distant organs at the time of diagnosis (1). Since NPC is highly sensitive to radiation, the most common and effective modern treatment of NPC patients is primarily based on radiotherapy. Although the local control rate of NPC is approaching 90\%, there are still $30-40 \%$ of NPC patients at the advanced stage subsequently developing distant metastases and/or local recurrences (2). More importantly, most of the mortalities are associated with these secondary metastases of NPC found in distant organs, such as the liver and lung. Once metastasis occurs, the prognosis of patients is poor, with a median survival of less than 12 months (3). Currently, there is no effective treatment for metastasis of NPC and, therefore, novel strategies are urgently needed.

As an essential etiological factor, Epstein-Barr virus (EBV) is closely related to NPC. The EBV genome is present virtually in all NPC cells (4). More interestingly, EBV-positive malignancies are usually associated with a state of persistent latent infection (5). EBV infection in NPC cells is classified as latency type II, in which only a few latent genes are expressed, such as Epstein-Barr nuclear antigen-1 (EBNA-1), latent 
membrane protein-1 (LMP-1), and EBV-encoded RNAs (EBERs) (6). Among these gene products, LMP-1 is considered to play a critical role in the development of NPC.

LMP-1 is a $63-\mathrm{kDa}$ integral membrane protein comprising a short $\mathrm{N}$-terminal domain, six transmembrane domains, and a 200 amino acid $\mathrm{COOH}$-terminal domain (7). As a viral oncogene, LMP-1 has been reported to be a useful target to screen for NPC (8). Although LMP-1 were detected in only $60 \%$ of invasive NPCs, all pre-invasive NPCs that quickly developed into invasive NPCs were LMP-1-positive (9). The expression of LMP-1 in NPC clinical patients suggests that LMP-1-positive NPC cells have a more progressive manner and a higher tendency to metastasize than LMP-1-negative NPC cells (10). A recent report has shown that LMP-1 contributes to multiple aspects of NPC metastasis (11). It functions as a constitutively active tumor necrosis factor receptor engaging a multitude of signaling pathways including NF-кB, MAPKs, JNK, p38, the JAK/STAT pathway and, more recently, the small Rho GTPases (12). It also influences other oncogenes involved in tumor metastasis, such as matrix metalloproteinases (MMPs) $(13,14)$. However, the effect of suppressing LMP-1 in the growth and metastasis of NPC in vivo remains to be demonstrated.

Investigation of EBV in NPC cells has been limited by the fact that most established NPC cell lines do not harbor EBV or lose it after long-term culture (15). C666-1 cells, a well-established NPC cell line, have been shown to be able to express EBV genome consistently and can express EBNA-1 protein, LMP-1 and -2 transcripts, and thus resemble the EBV latency II pattern (14). Furthermore, we have recently shown that $\mathrm{C} 666-1$ has high metastatic potential and suppression of LMP-1 causes reduction of metastatic potential in C666-1 cells in vitro (16). Thus, C666-1 provides a good cell model for NPC metastasis research. Since no NPC animal metastasis model has been reported so far, we have established a nude mouse model with which C666-1 NPC xenograft was transplanted in the liver and then metastasized to the lung.

In this study, we used a recombinant adeno-associated virus serotype 2 vector (rAAV-2) to deliver small hairpin RNA (shRNA) targeting EBV LMP-1, and evaluated the effects of long-term suppression of LMP-1 on NPC growth and metastasis in vivo. We found that suppression of LMP-1 in C666-1 cells using recombinant AAV to deliver shRNA targeting LMP-1 can significantly inhibit metastasis in vivo and in vitro. However, such suppression has no effect on C666-1 cell and NPC xenograft growth.

\section{Materials and methods}

Cell lines and culture conditions. The EBV-positive NPC cell line, C666-1, was kindly provided by Dr Dolly P. Huang from the Chinese University of Hong Kong. This cell line was cultured in RPMI-1640 (Invitrogen) with 10\% fetal bovine serum (FBS; Gibco BRL, Grand Island, NY). For virus packaging, HEK-293FT cells were obtained from American Type Culture Collection (Manassas, VA) and cultured in DMEM (Invitrogen) with 10\% FBS.

Preparation of $r A A V$-shRNA-LMP-1 and $r A A V$-EGFP. Plasmid pAAV-shRNA-LMP-1 was constructed by inserting shRNA targeting EBV LMP-1 (Fig. 1A) into the multiple cloning site of rAAV-2 vector (Fig. 1B). Plasmid pAAV-EGFP was constructed similarly by inserting full-length enhanced green fluorescent protein (EGFP) gene between Xho I and EcoR I sites of AAV-2 vector. Recombinant AAV (rAAV) particles were produced using a helper virus-free system as previously described (17-19), with minor modifications. Briefly, rAAV vectors and helper plasmid pDG were co-transfected into HEK-293FT cells by calcium phosphate precipitation method. Transfected cells were harvested at $60 \mathrm{~h}$ after transfection. They were trypsinized, and re-suspended in Tris-buffer $\mathrm{pH}$ 8.0. After two cycles of freezing/thawing, they were centrifuged for $20 \mathrm{~min}$ at $18000 \mathrm{x} \mathrm{g}$ (Bechman Avanti $\left.{ }^{\mathrm{TM}} \mathrm{J}-25 \mathrm{I}\right)$. The supernatants containing either rAAV-shRNA-LMP-1 or rAAV-EGFP particles were collected. rAAV particles were purified by HiTrap Heparin column chromatography (Sigma, St. Louis, MO). Peak virus fractions were collected and dialyzed against PBS containing $1 \mathrm{mM} \mathrm{MgSO}_{4}$. Viral solutions were then concentrated using a 100K-MicroSep Centrifugal Concentrator (Life Technologies, Carlsbad, CA), and viral titer was quantified by real-time PCR using the Taqman Universal PCR kit (Applied Biosystems), with a forward primer, 5'-CGGCTGTTGGGCACTGA-3', and a reverse primer, 5'-CCGAAGGGACGAAGCAGAAG-3'. Aliquots of viral stocks $\left(2 \times 10^{12} \mathrm{v} . \mathrm{g} . / \mathrm{ml}\right.$ each $)$ were stored at $-80^{\circ} \mathrm{C}$ until ready for use.

rAAV mediated transgene expression in vitro in C666-1 cells. C666- 1 cells $\left(2 \times 10^{5}\right)$ were seeded onto $35-\mathrm{mm}^{2}$ culture dishes. After $12 \mathrm{~h}$, medium was discarded and cells were infected with $5 \times 10^{4}$ rAAV-EGFP or rAAV-shRNA-LMP-1 viral particles per cell in RPMI-1640 for $10 \mathrm{~h}$. Then cells were gently washed twice with PBS, and fresh RPMI-1640 medium containing $10 \%$ FBS was added afterward. At $48 \mathrm{~h}$ post infection (pi), expression of EGFP was confirmed by fluorescent microscope (Olympus, TH4-200, x100). Three fields were randomly selected and the mean infection efficiency of rAAV-EGFP was calculated. Expression of LMP-1 at the RNA level was detected by RT-PCR. Total RNA of infected cells, PBS, rAAV-EGFP or rAAV-shRNA-LMP-1, was extracted for RT-PCR (Qiagen One-Step RT-PCR Mid Kit) following the protocol previously reported (16). GAPDH was chosen as a loading control (internal reference). RT-PCR products were visualized by agarose gel electrophoresis, and the relative mRNA level was determined using Gel-Pro Analyzer software.

Animal model of NPC xenograft transplanted in liver and metastasized to lung. Male nude mice (BALB/c-nu-/nu, 4-6 weeks old, weighing 18-22 g) were obtained from Nanfang Medical University (Guangzhou, China). C666-1 cells infected with rAAV-EGFP or rAAV-shRNA-LMP-1 were harvested at $48 \mathrm{~h}$ pi. Cells without infection were used as parallel control. All animals were maintained under specific pathogen-free conditions. Mice were anaesthetized with $1 \%$ pentobarbital sodium $(40 \mathrm{mg} / \mathrm{kg}$ ) before surgery. Primary tumor was inoculated by direct injection of $2 \times 10^{6}$ non-infected or infected C666-1 cells into the liver via laparotomy under direct visualization. All mice were divided into three groups (control, rAAV-EGFP, and rAAV-shRNA-LMP-1), and each group contained 12 mice. After 14 days, half of the mice from each group were sacrificed. 
Tumor volume of mice was recorded and metastasis in the liver and lung was observed. The rest of the mice were used to carry out a survival study, the number of mice that survived in each group was recorded everyday and a survival curve was constructed and analyzed by a log-rank test. The experiments were repeated once with reproducible results.

Histology. To evaluate the tumor volume and degree of tumor metastasis, the livers and lungs of treated mice were excised, fixed in $4 \%$ paraformaldehyde, and then paraffin-embedded. Tissue sections were stained with haematoxylin and eosin (H\&E). The long (a) and short (b) diameters of the xenografted tumor were measured, and tumor volume was calculated as $\mathrm{ab}^{2} \times 0.52$.

Immunohistochemical analysis. Tissues from paraffin blocks were sectioned, de-waxed and hydrated. Antigens were retrieved by heating in the microwave. Sections were then treated with $3 \%$ hydrogen peroxide for $10 \mathrm{~min}$, and incubated with anti-MMP-9 primary antibody (MaiXin Ltd., FuZhou, P.R. China) for $1 \mathrm{~h}$. After washing with PBS, they were further incubated with HRP-conjugated secondary antibody (MaiXin Ltd) for $30 \mathrm{~min}$ and stained with DAB solution. Results were evaluated under a light microscope. Fifteen fields under magnification x400 were randomly chosen for each specimen. Total number of positive cells in 15 fields was counted and divided by the average number of positive cells in each field, and the result was subjected to a grading system: negative (-), no positive cell; weak positive (+), number of positive cells $<3$; medium positive $(++)$, number of positive cells between 3 and 6 ; strong positive $(+++)$, number of positive cells $>6$.

MTT assay. MTT assay was performed to elucidate the effect of LMP-1 on cell proliferation. C666- 1 cells $\left(5 \times 10^{3}\right.$ per well) were plated onto 96-well plates. After 12-h incubation, medium was discarded and cells were infected with $5 \times 10^{4}$ rAAV-EGFP or rAAV-shRNA-LMP-1 viral particles per cell in RPMI-1640. An equal volume of medium was added to the non-infected control. Six replicates were performed for each group. Ten hours pi, fresh RPMI-1640 medium containing 10\% FBS was added to replace the old medium. At 24,48 and $72 \mathrm{~h} \mathrm{pi}$, $20 \mu 1$ of MTT reagent $(5 \mathrm{mg} / \mathrm{ml}$; Sigma) was added to each well, and cells were incubated for another $4 \mathrm{~h}$ at $37^{\circ} \mathrm{C}$. After incubation, the medium was discarded and replaced by $150 \mu \mathrm{l}$ of DMSO (Sigma) in each well. Samples were thoroughly mixed for $30 \mathrm{~min}$, and UV-absorbance at $570 \mathrm{~nm}$ was detected.

Flow cytometry. C666-1 cells infected with rAAV-EGFP or rAAV-shRNA-LMP-1 were collected at $48 \mathrm{~h}$ pi, and fixed in $70 \%$ ethanol at $4^{\circ} \mathrm{C}$ for $24 \mathrm{~h}$. After fixation, cells were resuspended in PBS, digested with RNase A (Sigma) at $37^{\circ} \mathrm{C}$ for $30 \mathrm{~min}$, and then incubated with propidium iodide (Sigma) for $20 \mathrm{~min}$. Five thousand cells from each sample were analyzed by flow cytometry (Coulter Epics, XL, UK). Four replicates were performed for each group. The cell cycle phase distribution was calculated from the resultant DNA histogram using Multicycle AV software (Phoenix Flow System, San Diego, CA, USA).
Wound healing assay. Wound healing assay was carried out to determine the ability of cells to form membrane protrusion and cell migration. C666- 1 cells $\left(2 \times 10^{5}\right.$ per well) were seeded onto 6-well plates. Cells were infected with $5 \times 10^{4}$ rAAVEGFP or rAAV-shRNA-LMP-1 viral particles per cell in RPMI-1640. Non-infected cells were used as a parallel control in this experiment, and four replicates were performed for each group. At $10 \mathrm{~h}$ pi, fresh RPMI-1640 medium containing $10 \%$ FBS was added to replace the old medium. At $48 \mathrm{~h}$ pi, a single wound was created in the centre of the cell monolayer by gentle removal of the attached cells with a sterile plastic pipette tip. The debris was removed by washing the cells with serum medium. Migration of cells into the wound was then observed at different time points (2, 4, 6, 8 and $10 \mathrm{~h})$. Cells which migrated into the wounded area or protruded from the border of the wound were visualized and photographed under an inverted microscope (Leica, German). A total of 10 areas were selected randomly in each well under a 100x objective, and cells (in 4-wells) of each group were quantified in each experiment.

In vitro Matrigel invasion assay. Cell invasiveness was determined in vitro by the ability of the cells to transmigrate a layer of extracellular matrix in Matrigel in Biocoat Matrigel Invasion chamber (Becton-Dickenson Labware, Bedford, MA). C666-1 cells ( $1 \times 10^{5}$ per well) were seeded onto 12-well plates. Cells were infected with $5 \times 10^{4}$ rAAV-EGFP or rAAV-shRNALMP-1 viral particles per cell in RPMI-1640, and followed by replacement of fresh RPMI-1640 medium containing $10 \%$ FBS after 10-h infection. At $48 \mathrm{~h}$ pi, cells were collected and plated at a density of $2 \times 10^{4}$ cells/insert. Medium with $10 \%$ FBS was added to the lower chamber as a chemoattractant. After incubation for $22 \mathrm{~h}$, cells on the upper surface of the membrane were removed. Invasive cells on the lower surface were fixed in $100 \%$ methanol and stained with $1 \%$ Toluidine (Sigma). The number of invasive cells was counted in three random optical fields (x40 and x200) under an inverted microscope (Leica). Experiments for each individual sample were performed in triplicate chambers and were repeated.

Statistical analysis. One way ANOVA and independent t-test were employed for statistical analysis and Kruskal-Wallis test was used for immunohistochemical analysis. All data were analyzed by SPSS10.0 software (SPSS Inc. SPSS Advanced models 10.0, Chicago, IL, USA), with $\mathrm{P}<0.05$ considered statistically significant.

\section{Results}

Recombinant $A A V$ infection efficiency and inhibition of LMP-1 by rAAV-shRNA-LMP-1 in vitro. C666-1 cells were infected with rAAV-EGFP in a dose-dependent manner of $5 \times 10^{2}, 5 \times 10^{3}, 1 \times 10^{4}$, or $5 \times 10^{4}$ v.g./cell, and the expression of recombinant protein was confirmed at $10 \mathrm{~h}$ pi using a fluorescent microscope. Maximum fluorescent signal was observed at $48 \mathrm{~h}$ after infection (Fig. 1C). Highest infection efficiency $(\sim 95 \%)$ was obtained at $48 \mathrm{~h}$ pi with a multiplicity of infection of $5 \times 10^{4} \mathrm{v} . \mathrm{g} . / \mathrm{cell}$ (Fig. 1D). Suppression of LMP-1 expression in rAAV-shRNA-LMP-1 (5x10 4 v.g./cell) infected C666-1 cells was evaluated by RT-PCR at $48 \mathrm{~h}$ pi 
A.

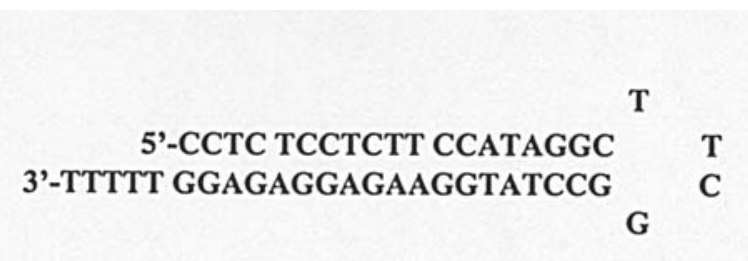

B.

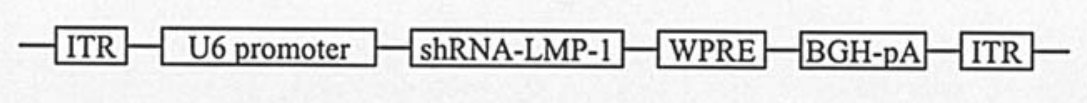

C.
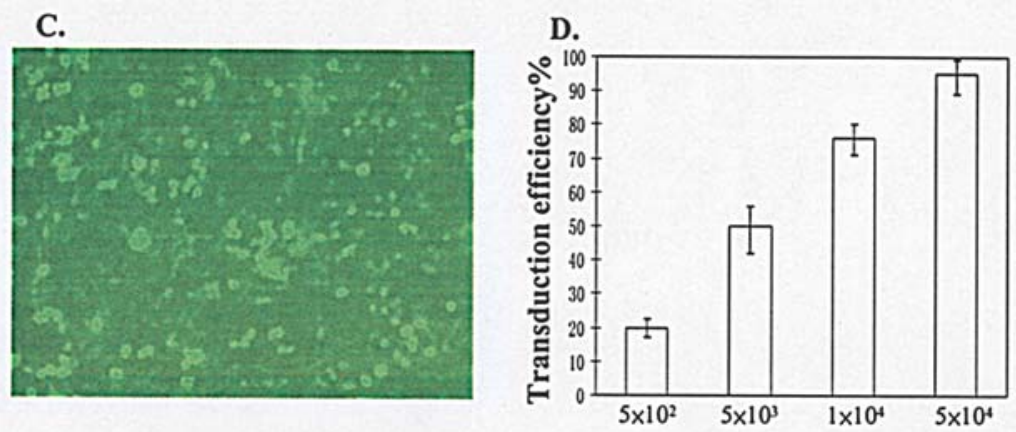

E.

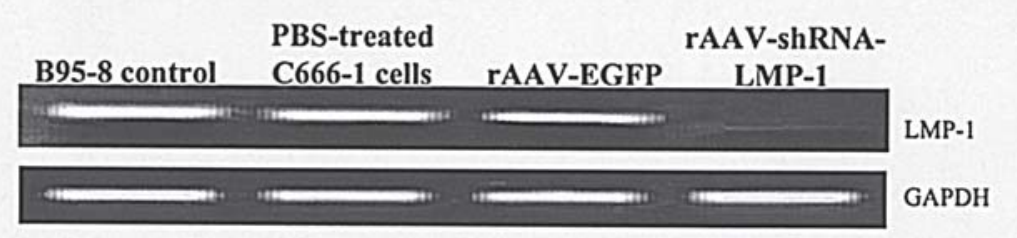

Figure 1. Infection of C666-1 cells by rAAV viruses. (A) shRNA sequence targeting EBV encoded LMP-1; (B) rAAV-shRNA-LMP-1 plasmid construction; (C) infection of C666-1 cells with rAAV-EGFP at a MOI of $5 \times 10^{4}$ for $48 \mathrm{~h}$ was observed under fluorescent microscope (x100); (D) optimal infection efficiency of C666-1 cells with rAAV-EGFP obtained at MOI of 5x104 (cells were incubated for $48 \mathrm{~h}$ ); (E) RT-PCR showed that expression of LMP-1 in RNA level was effectively suppressed by rAAV-shRNA-LMP-1 but not by rAAV-EGFP infection.

(Fig. 1E). B95-8, EBV transformed marmoset B lymphocyte, was used as a parallel positive control. Results demonstrated that rAAV-shRNA-LMP-1 effectively and actively suppressed the expression of LMP-1 in the infected C666-1 cells.

Establishment of NPC metastasis nude mice model and examine the effect of $r A A V$-shRNA-LMP-1 treatment on survival rate. A NPC xenografted nude mice model $(\mathrm{n}=36)$ was established. Primary tumors were inoculated by direct injection of $2 \times 10^{6}$ C666- 1 cells into the livers as a single nodule. Five days after inoculation, 12 mice were sacrificed, and primary tumors were successfully formed in the livers of all the nude mice. At day 10 post inoculation, another 12 mice were sacrificed. Other than the single large round primary liver tumors found at the original injection sites, there are obvious metastasis in other parts of the livers, with approximately two third of these mice developed lung metastasis. High mortality rate of the remaining 12 nude mice was found as half of the mice $(n=6)$ died at around 14 days after inoculation due to advanced tumor growth and metastasis.

To determine whether suppression of LMP-1 by rAAVshRNA-LMP-1 can prolong survival and reduce the tumor size of NPC xenografted nude mice, we harvested three groups of C666-1 cells, which were non-infected and infected with either rAAV-EGFP or rAAV-shRNA-LMP-1. Primary tumors

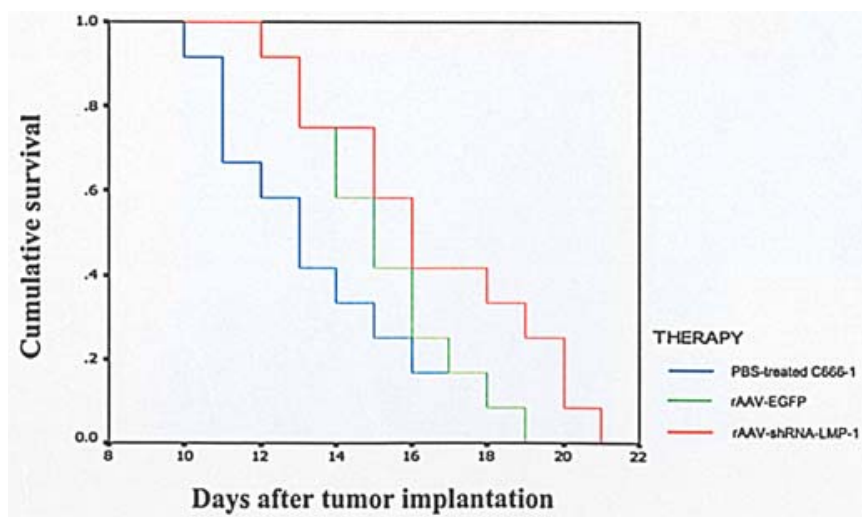

Figure 2. rAAV-shRNA-LMP-1 treatment could not prolong the survival of NPC-bearing mice. Survival study for xenograft NPC nude mice. Primary tumors were established by direct injection of $2 \times 10^{6}$ of rAAV-shRNA-LMP-1 infected (red line), or rAAV-EGFP infected (green line), or PBS-treated C666-1 cells (blue line) into the livers. The survival rate was recorded everyday and a survival curve was constructed and analyzed by a log-rank test.

were established by direct injection of $2 \times 10^{6}$ cells from each group into the livers of three groups of mice $(n=12$ per group). Mice were checked for survival everyday. Although rAAV-shRNA-LMP-1 treatment showed a slightly increased 
Table I. Anti-tumor effects of rAAV-mediated rAAV-shRNA-LMP-1 therapy in NPC xenograft transplanted mice.

\begin{tabular}{lccccc}
\hline $\begin{array}{l}\text { Treatment } \\
\text { group }\end{array}$ & $\begin{array}{c}\text { No. of } \\
\text { mice }\end{array}$ & $\begin{array}{c}\text { Transplanted tumor } \\
\text { volume }\left(\mathrm{cm}^{3}, \bar{x} \pm \mathrm{SD}\right)\end{array}$ & $\begin{array}{c}\text { Metastases } \\
\text { in liver }(\%)\end{array}$ & $\begin{array}{c}\text { Metastases } \\
\text { to lung }(\%)\end{array}$ & $\begin{array}{c}\text { No. of lung metastasis } \\
\text { nodules/animal }(\bar{x} \pm \text { SD) }\end{array}$ \\
\hline rAAV-shRNA-LMP-1 & 12 & $0.2527 \pm 0.1152$ & $50^{\mathrm{a}}(6 / 12)$ & $66(8 / 12)$ & $1.50 \pm 0.22^{\mathrm{b}}$ \\
rAAV-EGFP & 12 & $0.2533 \pm 0.0754$ & $100(12 / 12)$ & $100(12 / 12)$ & $3.67 \pm 0.67$ \\
PBS-treated C666-1 & 12 & $0.3163 \pm 0.1363$ & $100(12 / 12)$ & $100(12 / 12)$ & $2.83 \pm 1.03$ \\
\hline
\end{tabular}

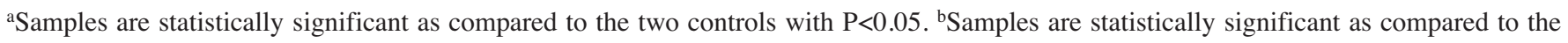
two controls with $\mathrm{P}<0.05$.

Table II. Down-regulation of expression of MMP-9 by antitumor metastasis of rAAV-shRNA-LMP-1 gene therapy in NPC xenograft transplanted mice.

\begin{tabular}{lcccccc}
\hline \multirow{2}{*}{ Group } & \begin{tabular}{c} 
No. of \\
random \\
\cline { 3 - 7 }
\end{tabular} & \multicolumn{4}{c}{ Infiltrate intensity } \\
\cline { 3 - 6 } & fields & +++ & ++ & + & - \\
\hline C666-1-rAAV-LMP-1 $^{\text {a }}$ & 15 & 0 & 8 & 1 & 6 \\
C666-1-rAAV-EGFP & 15 & 1 & 9 & 4 & 1 \\
PBS-treated C666-1 & 15 & 6 & 6 & 3 & 0 \\
\hline
\end{tabular}

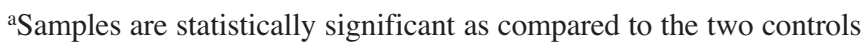
with $\mathrm{P}<0.05$.

survival rate (Fig. 2), the median survival of the rAAV-shRNALMP-1 treatment group (16.50 \pm 3.06 days) was not found to be significantly longer than that of the two controls: rAAVEGFP treatment group (15.20 \pm 2.12 days) and PBS-treated C666-1 group (13.60 \pm 2.91 days).

rAAV-shRNA-LMP-1 inhibits tumor metastasis but not tumor growth in vivo. At day 14 post C666-1 cell inoculation, mice in the above three groups were sacrificed ( $n=6$ per group). The anti-metastasis effect of the rAAV-shRNA-LMP-1 was also examined. All of the transplanted tumors appeared in round shape, and grew on the surface of the livers (Fig. 3A-C, dashed line circles). All livers of the control rAAV-EGFP treatment (Fig. 3B) and PBS-treated C666-1 (Fig. 3C) groups displayed multiple metastasized tumors of different sizes on the surface (blue arrows). By contrast, half of the rAAVshRNA-LMP-1 treated mice showed no sign of liver metastasis and only the primary tumors were found (Fig. 3A and Table I), and half of the mice showed a significant reduction in the number of metastasized tumors.

To explore the effect of rAAV-shRNA-LMP-1 in inhibiting distant metastasis, we evaluated the morphology of the lungs from the three groups of mice. There was a reduction of metastases in the lungs of rAAV-shRNA-LMP-1 treated mice, with one third of these mice showing normal lung morphology without any obvious indication of metastasis nodule (non-metastasis) and the remaining mice showing a reduction in number and size of lung metastasis nodules (less metastasis) (Fig. 3D and Table I). Whereas the lungs from the two control groups, rAAV-EGFP (Fig. 3E) and PBStreated C666-1 (Fig. 3F) groups, showed heavy metastasis and multiple lung metastasis nodules (Table I).

In the rAAV-shRNA-LMP-1 treated mice with lung metastasis, pathological sections revealed the number of lung metastasis nodules to be $1.5 \pm 0.22$, which was statistically significant as compared to $3.67 \pm 0.67$ and $2.83 \pm 1.03$ in the rAAV-EGFP treatment and PBS-treated C666-1 control groups, respectively (Table I). Distant metastasis was also confirmed using histology. H\&E staining revealed that there was no sign of metastasized tumor in lung tissues of the same non-metastasis rAAV-shRNA-LMP-1 treated mice (Fig. 4D), whereas histological studies confirmed the presence of metastasized tumors in the lung tissues from the rAAVEGFP treatment (Fig. 4E) and PBS-treated C666-1 (Fig. 4F) groups. Collectively, our data suggest that rAAV-shRNALMP-1 significantly inhibited distant metastasis in the NPC xenograft nude mice.

The volume of the primary liver tumors was not found, however, to be significantly different among the three groups (Table I). In addition, histological studies of the liver tumors did not detect differences in the primary implanted tumors among all groups (Fig. 4A-C). For the in vitro study, we detected the effect of suppression of LMP-1 on C666-1 cell growth by MTT assay and cell cycle by flow cytometry. Our data showed that suppression of LMP-1 by rAAV-shRNALMP-1 had no effect on cell growth and cell cycle in the infected cells (data not shown).

Suppression of LMP-1 by rAAV-shRNA-LMP-1 down-regulated the expression of $M M P-9$. Immunohistochemical detection of MMP-9 in the tumor tissues indicated that this protein was dominantly immunolocalized at the membrane and cytoplasm of the tumor cells (Fig. 5A-C). Fifteen fields were randomly selected to evaluate and grade for the expression of MMP-9 (Fig. 5D and Table II). The results demonstrated that the expression of MMP-9 was significantly lower in the rAAVshRNA-LMP-1 treatment group as compared to the control rAAV-EGFP treatment or PBS-treated C666-1 groups $(\mathrm{P}<0.01)$. This data strongly suggested that the suppression of LMP-1 in tumor cells effectively inhibited tumor metastasis through down-regulation of MMP-9.

Suppression of LMP-1 by rAAV-shRNA-LMP-1 infection decreased cell mobility and the ability for Matrigel trans- 


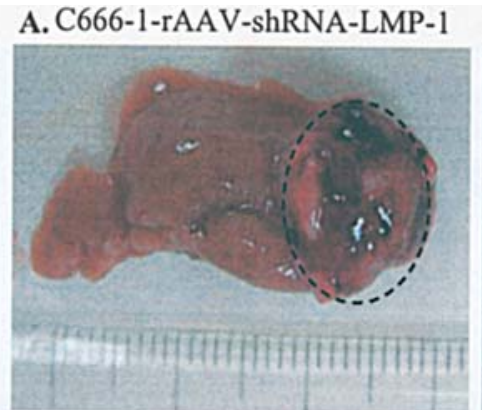

D.

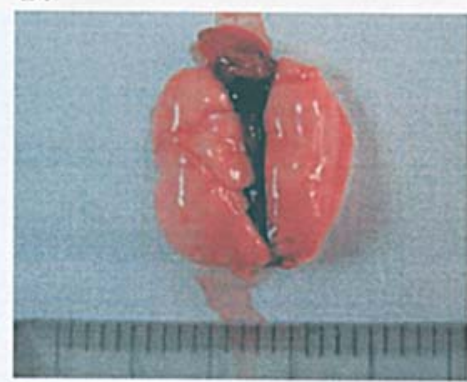

B. C666-1-rAAV-EGFP

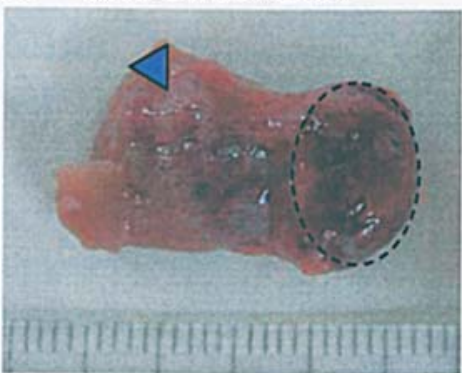

E.

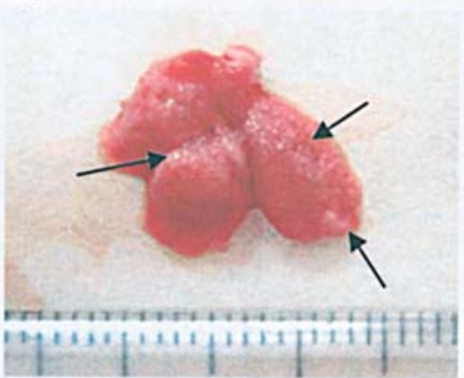

C. C666-1-PBS-treated

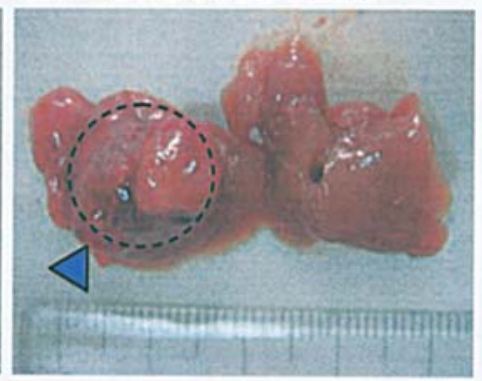

F.

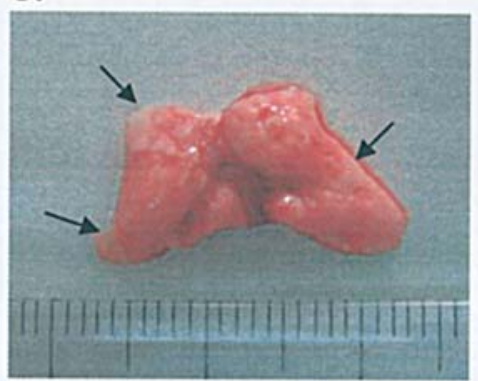

Figure 3. Gene therapy with rAAV-shRNA-LMP-1 treatment prevents liver and lung metastasis. Photographs taken from nude mice at day 14 after tumor inoculation. Liver morphology was examined from each of the treatment groups: (A) rAAV-shRNA-LMP-1; (B) rAAV-EGFP; and (C) PBS-treated C666-1. Dashed-line circles indicate the sites of the primary NPC tumors inoculated. Blue arrows indicate the signs of metastasis in rAAV-EGFP and C666-1 cells group. Lung morphology at day 14 after tumor inoculation: (D) rAAV-shRNA-LMP-1 treated group; (E) rAAV-EGFP treated group; and (F) PBS-treated C666-1 group. Multiple tumor metastases were indicated by arrows in the two control groups.

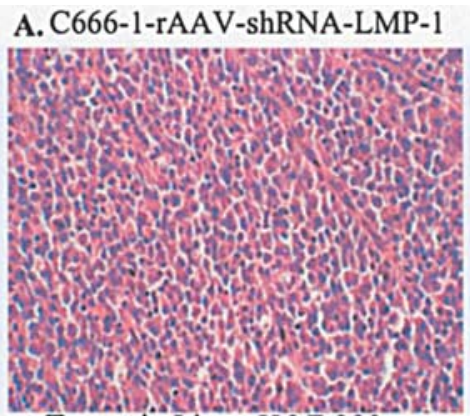

Tumor in Liver H\&E 200x

D. C666-1-rAAV-shRNA-LMP-1

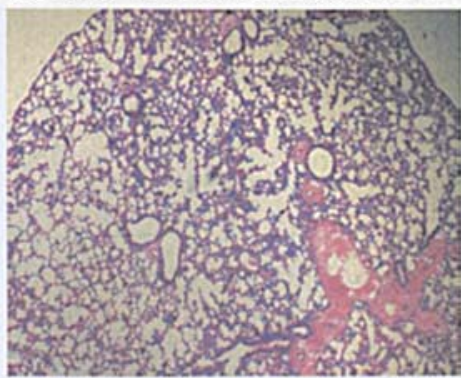

Lung H\&E 40x

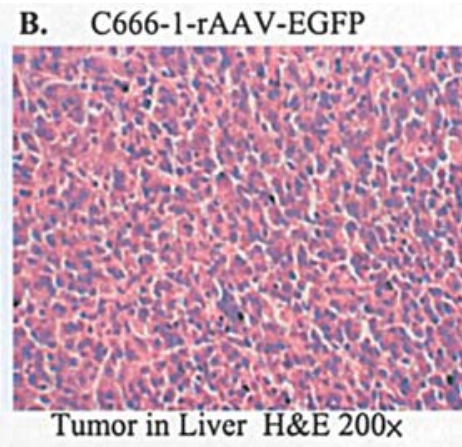

C. C666-1-PBS-treated

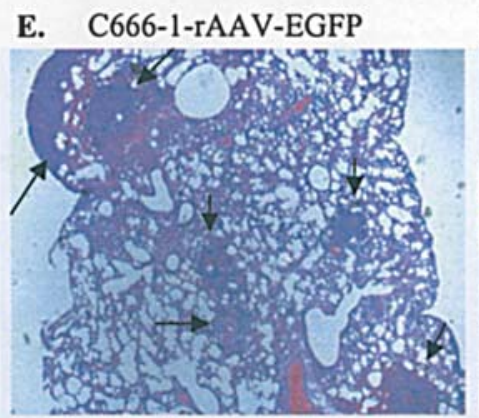

Lung H\&E 40x

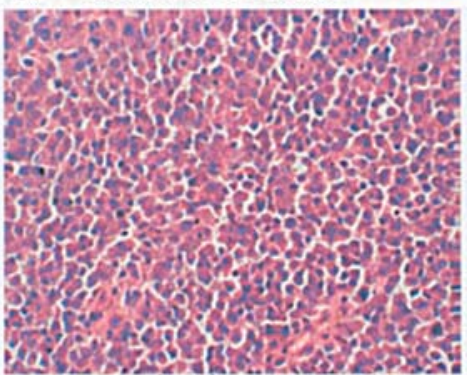

Tumor in Liver H\&E 200x

F. C666-1-PBS-treated

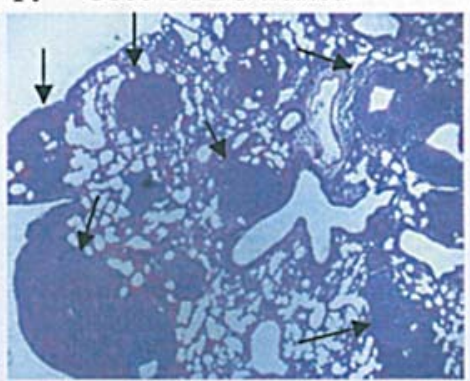

Lung H\&E 40x

Figure 4. rAAV-shRNA-LMP-1 treatment showed no effect on development of the primary transplanted tumors but prevented lung metastasis in the NPC xenograft nude mice. Tissue sections of formalin-fixed, paraffin-embedded primary tumors were stained by H\&E. Primary liver tumor tissues showed no different sign among the three groups: (A) rAAV-shRNA-LMP-1 treated group; (B) rAAV-EGFP treated group; and (C) PBS-treated C666-1 group. Lung tissues from: (D) rAAV-shRNA-LMP-1 treated mice; (E) rAAV-EGFP treated mice; and (F) PBS-treated C666-1 mice. Arrows indicate tumors that formed in metastasized lung tissues.

membrane migration. It has been reported that MMP-9 is markedly associated with invasion and metastasis of tumor cells $(20,21)$, and promotes tumor metastasis by selectively degrading type IV collagen (22). To evaluate active cell motility and invasion of basement membrane by tumor cells, we first measured the motility of the cells by wound healing assay. 


\section{A. C666-1-rAAV-shRNA-LMP-1}

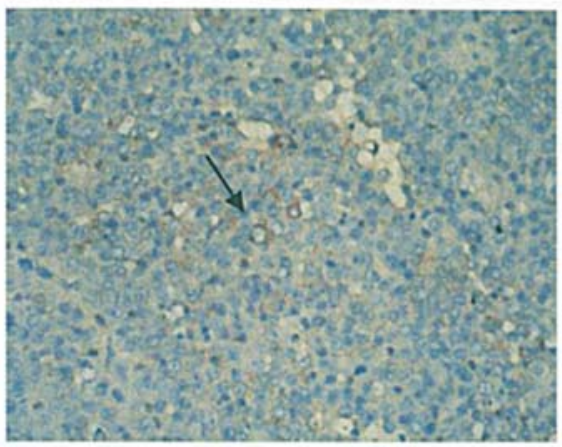

$400 x$

\section{C666-1-PBS-treated}

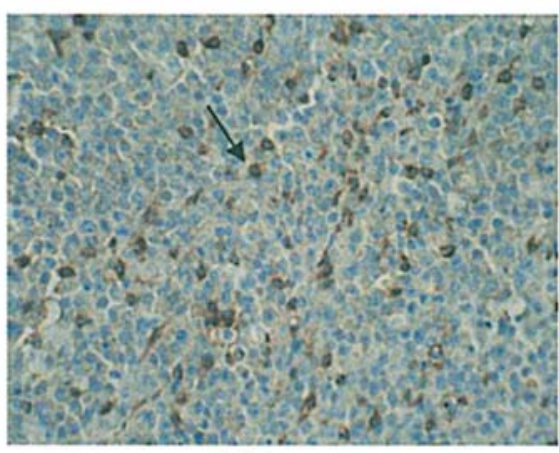

$400 x$

\section{B. C666-1-rAAV-EGFP}

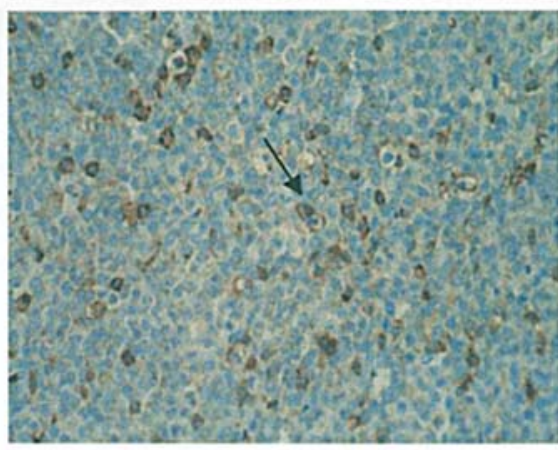

$400 x$

D.

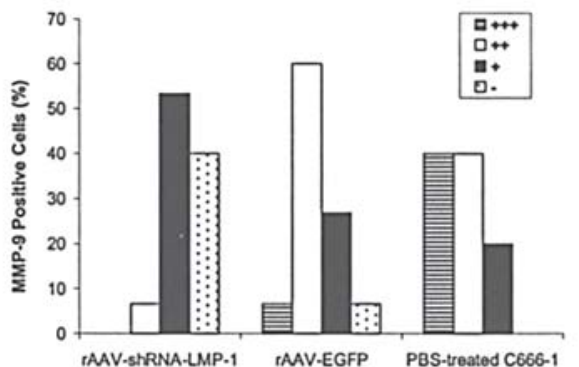

Figure 5. Down-regulation of expression of MMP-9 in rAAV-shRNA-LMP-1 treatment group. Immunohistochemical detection of MMP-9 in tumor tissue indicated that this protein was down-regulated when LMP-1 expression was suppressed. MMP-9 expression was quantitated in each of the three groups: (A) rAAV-shRNA-LMP-1 treatment group; (B) rAAV-EGFP treated group; and (C) PBS-treated C666-1 group. Summary of the number of MMP-9-positive cells was plotted as shown in (D), the number of MMP-9-positive cells was found to be statistically significantly decreased $(\mathrm{P}<0.01)$.
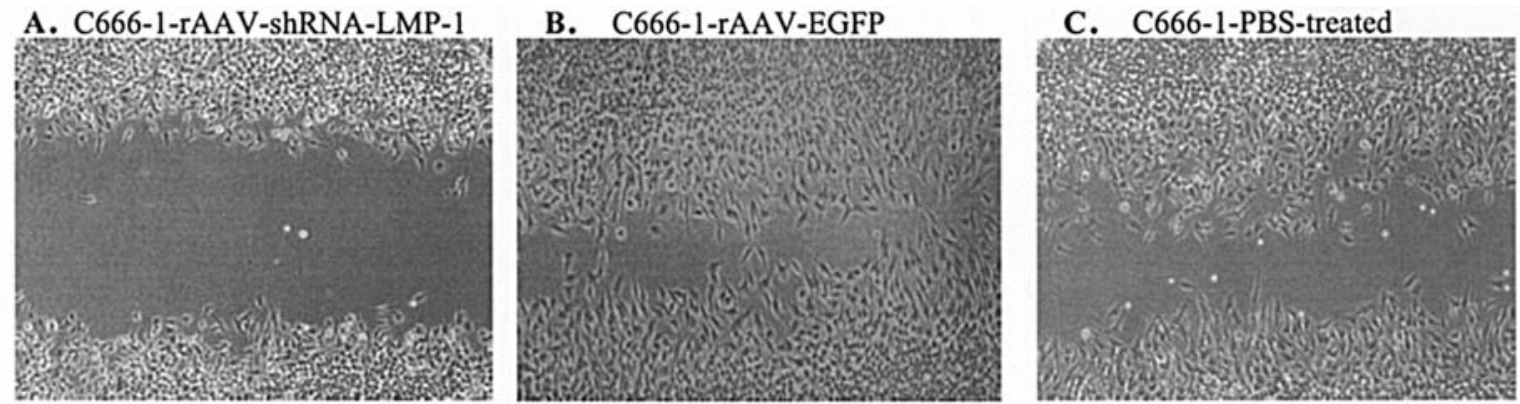

D.

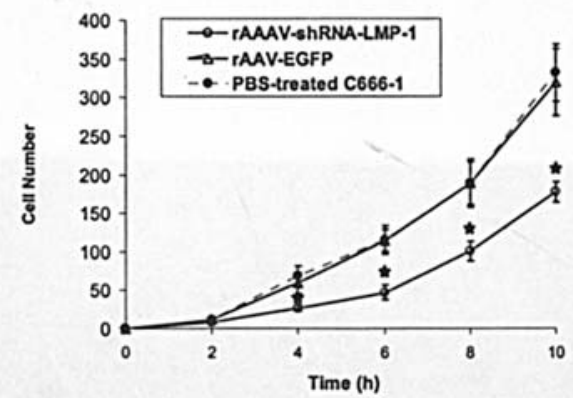

Figure 6. Suppression of LMP-1 expression by rAAV-shRNA-LMPreduced migration of C666-1 cells. At $8 \mathrm{~h}$ after wound formation, a large number of cells moved into the wound in rAAV-EGFP treated (B) and PBS-treated C666-1 groups (C); however, much less cells moved into the wound in the rAAV-shRNA-LMP-1 treated group (A). (D) A migration curve was plotted against each treatment group; asterisks indicate a statistically significant difference among the three groups in migration patterns $(\mathrm{P}<0.01)$.

As shown in Fig. 6D, as early as $4 \mathrm{~h}$ after wound formation, the rAAV-shRNA-LMP-1 infected cells displayed significantly lower cell invasion activity as compared to the control rAAV-
EGFP and PBS-treated C666-1 groups. At $8 \mathrm{~h}$ after wound formation, the two control groups had fully migrated towards the open wound (Fig. 6B and C). In contrast, the rAAV- 
A. C666-1-rAAV-shRNA-LMP-1

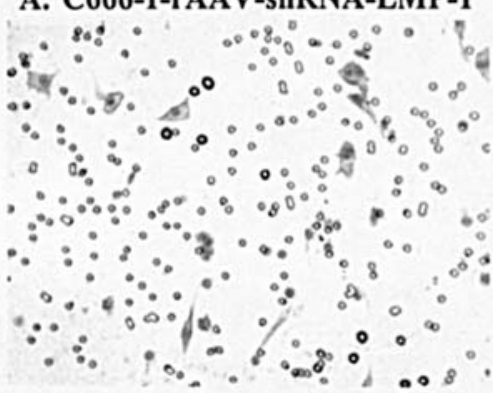

$200 x$
B. C666-1-rAAV-EGFP

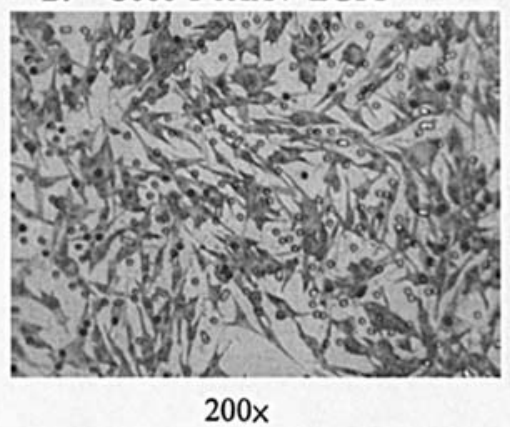

C. C666-1-PBS-treated

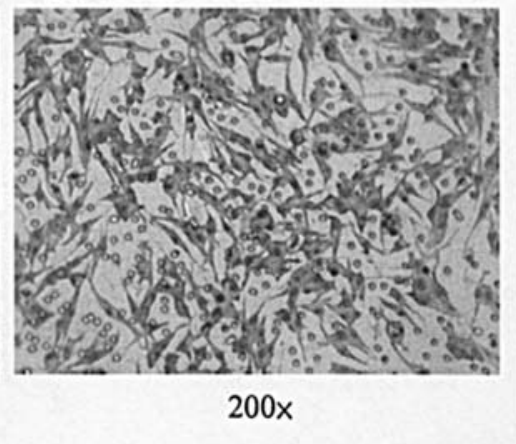

D.

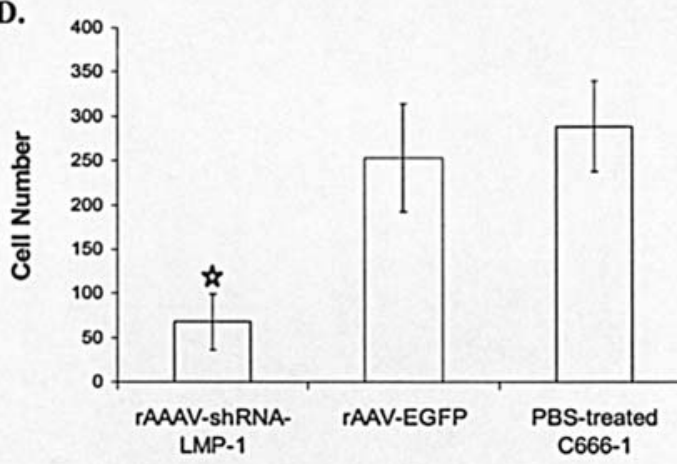

Figure 7. Suppression of LMP-1 expression hampered the transmembrane migration ability of C666-1 cells. A layer of Matrigel matrix occluded $8 \mu \mathrm{m}$ membrane pores and served as a reconstituted basement membrane. Invasive NPC cells were able to detach themselves from and invade through the Matrigel matrix and pores. As compared with the two controls, rAAV-EGFP treated C666-1 (B) and PBS-treated C666-1 cells (C), the invasive ability of rAAV-shRNA-LMP-1 treated C666-1 cells (A) showed a reduction of migrated cells by $>50 \%(P<0.01)$. (D) The number of migrated cells is presented in graphic form; asterisks indicate a statistically significant difference of the rAAV-shRNA-LMP-1 treatment group from the two control groups.

Metastasis is a phenomenon composed of sequential cascades involving multiple host-tumor interactions. Reduction of tumor cell adhesion and degradation of extracellular matrix (ECM) and basement membrane are thought to be the essential steps (14), in which neoplastic cells leave the primary lesion and invade local and distant host tissues $(23,24)$. It has been shown that LMP-1 can enhance cell motility through Ets-1 and c-Met activation (10), and also induces the gene expression of Matrix Metalloproteinases-9 (MMP-9) through activation of NF- $\mathrm{KB}$ and AP-1 by two carboxyl-terminal cytoplasmic domains (CTAR-1 and CTAR-2) $(9,25,26)$. MMP-9, a zinccontaining endopeptidase, is capable of degrading all kinds of extracellular matrix proteins, is secreted mainly by tumor cells and has been detected in a large variety of cancers, such as lung, prostate, breast, colon, and head and neck carcinomas (27-31). It has been reported that MMP-9 is markedly associated with invasion and metastasis of tumor cells $(20,21)$. It promotes tumor metastasis by selectively degrading type IV collagen, which is a major component of ECM (22). Therefore, the effect of LMP-1 to expression of MMP-9 was also investigated in this study. Our results indicated that expression of MMP-9 was down-regulated effectively after suppression of LMP-1 in vivo, which suggested that LMP-1 triggers metastasis in NPC cells at least in part through regulation of expression of MMP-9.

Recently, LMP-1 was reported to be detected in $100 \%$ of preinvasive NPCs (32). Therefore, it is a good target for gene therapy in studying NPC metastasis. Currently, there are few effective treatments available for NPC patients at the stage of metastasis. Our results provide preliminary data for the future development of a cocktail therapy which will include radiotherapy and long-term RNA interference therapy on LMP-1 mediated by AAV and/or other gene delivery systems for the prevention and treatment of NPC metastasis. a better gene therapy system for treating NPC diseases. 


\section{Acknowledgements}

We thank Professor Dolly Huang from the Chinese University of Hong Kong for kindly providing the C666-1 cell line, and Dr Rory Watt from the University of Hong Kong for critically reading this manuscript. This work was supported by the Guangdong Natural Science Fund, China (04020406, to X.-P. Li and Y. Peng), the National Natural Science Fund of China (30471945 to X.-P. Li), and in part by the Science and Technology Program of Guangdong province, China (2003c30303 to X.-P. Li and Y. Peng). This work is also supported by Li Ka Shing Institute of Health Sciences and grants from the Research Grant Council, Hong Kong, China (CUHK7422/03M to H.-F.K and HKU7243//02M to M.C.L.).

\section{References}

1. Hu LF, Chen F, Zheng X, et al: Clonability and tumorigenicity of human epithelial cells expressing the EBV encoded membrane protein LMP1. Oncogene 8: 1575-1583, 1993.

2. Teo PML and Chan ATC: Treatment strategy and clinical experience. Semin Cancer Biol 12: 497-504, 2002.

3. Ma BBY and Chan ATC: Recent perspectives in the role of chemotherapy in the management of advanced nasopharyngeal carcinoma. Cancer 103: 22-31, 2005.

4. Niedobitek G, Meru N and Delecluse HJ: Epstein-Barr virus infection and human malignancies. Int J Exp Pathol 82: 149-170, 2001.

5. Herrmann K and Niedobitek G: Epstein-Barr virus-associated carcinomas: facts and fiction. J Pathol 199: 140-145, 2003.

6. Pagano JS: The Epstein-Barr virus and nasopharyngeal carcinoma. Cancer 74: 2397-2398, 1994.

7. Fielding CA, Sandvej K and Mehl A: Epstein-Barr virus LMP-1 natural sequence variants differ in their potential to activate cellular signaling pathways. J Virol 75: 9129-9141, 2001.

8. Hao SP, Tsang NM and Chang KP: Screening nasopharyngeal carcinoma by detection of the latent membrane protein 1 (LMP-1) gene with nasopharyngeal swabs. Cancer 97: 1909-1913, 2003.

9. Horikawa T, Yoshizaki T, Sheen TS, Lee SY and Furukawa M: Association of latent membrane protein 1 and matrix metalloproteinase 9 with metastasis in nasopharyngeal carcinoma. Cancer 89: 715-723, 2000.

10. Horikawa T, Sheen TS, Takeshita H, Sato H, Furukawa M and Yoshizaki T: Induction of c-Met proto-oncogene by EpsteinBarr virus latent membrane protein-1 and the correlation with cervical lymph node metastasis of nasopharyngeal carcinoma. Am J Pathol 159: 27-33, 2001.

11. Yoshizaki T: Promotion of metastasis in nasopharyngeal carcinoma by Epstein-Barr virus latent membrane protein-1. Histol Histopathol 17: 845-850, 2002.

12. Tsao SW, Tramoutanis G, Dawson CW, Lo AK and Huang DP: The significance of LMP1 expression in nasopharyngeal carcinoma. Semin Cancer Biol 12: 473-487, 2002.

13. Yoshizaki T, Sato H, Furukawa M and Pagano JS: The expression of matrix metalloproteinase-9 is enhanced by Epstein-Barr virus latent membrane protein-1. Proc Natl Acad Sci USA 95: 3621-3626, 1998.

14. Stetler-Stevenson WG and Yu AE: Proteases in invasion: matrix metalloproteinases. Semin Cancer Biol 11: 143-152, 2001.
15. Cheung ST, Huang DP, Hui AB, et al: Nasopharyngeal carcinoma cell line (C666-1) consistently harbouring EpsteinBarr virus. Int J Cancer 83: 121-126, 1999.

16. Li XP, Li G, Peng Y, Kung HF and Lin MC: Suppression of Epstein Barr virus-encoded latent membrane protein-1 by RNA interference inhibits the metastatic potential of nasopharyngeal carcinoma cells. Biochem Biophys Res Commun 315: 212-218, 2004.

17. Xiao X, Li J and Samulski RJ: Role for highly regulated rep gene expression in adeno-associated virus vector production. J Virol 7: 5236-5243, 1997.

18. Grimm D, Kern A, Rittner K and Kleinschmidt JA: Novel tools for production and purification of recombinant adenoassociated virus vectors. Hum Gene Ther 9: 2745-2760, 1998.

19. Matsushita T, Elliger S, Elliger C, et al: Adeno-associated virus vectors can be efficiently produced without helper virus. Gene Ther 7: 938-945, 1998.

20. Himelstein BP, Canete SR, Bernhard EJ, Dilks DW and Muschel RJ: Metalloproteinase in tumor progression: the contribution of MMP-9. Invasion Metastasis 14: 246-258, 1994.

21. D'Errico A, Garbisa S, Liotta LA, Castronovo V, StetlerStevenson WG and Grigioni WF: Augmentation of type IV collagenase, laminin receptor, and Ki67 proliferation antigen associated with human colon, gastric, and breast carcinoma progression. Mod Pathol 4: 239-246, 1991.

22. Liotta LA: Tumor invasion and metastasis - role of the extracellular matrix: Rhoads memorial award lecture. Cancer Res 46: $1-7,1986$.

23. Fidler IJ and Hart IR: Biologic diversity in metastatic neoplasms origins and implications. Science 217: 998-1001, 1982.

24. Liotta LA, Tryggvason K, Garbisa S, Hart IR, Foltz CM and Shafie S: Metastatic potential correlates with enzymatic degradation of basement membrane collagen. Nature 284: 67-68, 1980

25. Huen DS, Henderson SA, Carter DC and Rowe M: The EpsteinBarr virus latent membrane protein 1 (LMP1) mediates activation of NF-kB and cell surface phenotype via two effector regions in its carboxy-terminal cytoplasmic domain. Oncogene 10: 549-560, 1995.

26. Takeshita H, Yoshizaki T, Miller WE, Sato H, Furukawa M and Pagano JS: Matrix metalloproteinase 9 expression is induced by Epstein-Barr virus latent membrane protein $1 \mathrm{C}$-terminal activation regions 1 and 2. J Virol 73: 5548-5555, 1999.

27. Tokuraku M, Sato H, Murakami S, Okada Y and Seiki M: Activation of the precursor of gelatinase A/72 kDa type IV collagenase/MMP-2 in lung carcinomas correlates with the expression of membrane-type matrix metalloproteinase (MTMMP) and with lymph node metastasis. Int J Cancer 64: 355-359, 1995

28. Wood M, Fudge K, Mohler JL, et al: In situ hybridization studies of metalloproteinases 2 and 9 and TIMP-1 and TIMP-2 expression in human prostate cancer. Clin Exp Metastasis 15: 246-258, 1997.

29. Rha SY, Kim JH, Roh JK, et al: Sequential production and activation of matrix-metalloproteinase-9 (MMP-9) with breast cancer progression. Breast Cancer Res Treat 43: 175-181, 1997.

30. Newell KJ, Witty JP, Rodgers WH and Matrisian LM: Expression and localization of matrix- degrading metalloproteinases during colorectal tumorigenesis. Mol Carcinog 10: 199-206, 1994.

31. Yoshizaki T, Sato H, Maruyama Y, et al: Increased expression of membrane type1-matrix metalloproteinase in head and neck carcinoma. Cancer 79: 139-144, 1997.

32. Pathmanathan RU, Prasad U, Sadler R, Flynn K and RaabTraub N: Clonal proliferations of cells infected with EpsteinBarr virus in preinvasive lesions related to nasopharyngeal carcinoma. N Engl J Med 333: 693-698, 1995. 